Ramos-Rodríguez, E. \& Reyes-Santander, P. (2017). Favoreciendo la reflexión del docente: un Estudio de Clases sobre cálculo integral usando tecnología. Revista Electrónica Interuniversitaria de Formación del Profesorado, 20(1), 67-85.

\title{
Favoreciendo la reflexión del docente: un Estudio de Clases sobre cálculo integral usando tecnología
}

\author{
Elisabeth Ramos-Rodríguez ${ }^{(1)}$, Pamela Reyes-Santander ${ }^{(2)}$ \\ ${ }^{(1)}$ Pontificia Universidad Católica de Valparaíso, Chile; ${ }^{(2)}$ Universidad de Bielefeld, Alemania
}

\section{Resumen}

Para enfrentar la pregunta ¿cómo fortalecer en un entorno colectivo, la reflexión sobre su práctica de profesores universitarios? diseñamos una alternativa de formación basada en una sesión de Estudio de Clases articulada con la noción de reflexión sistemática. Establecimos referentes teóricos desde la reflexión y la noción de tarea matemática para abordar, con un enfoque cualitativo, un estudio interpretativo. Examinamos los datos extraídos de transcripciones de la grabación en vídeo de la sesión realizada en un evento internacional. Se observa que la alternativa de formación propuesta presenta elementos que favorecen la reflexión sobre la práctica del docente.

\section{Palabras clave}

Reflexión docente; Estudio de Clases; cálculo integral; tecnología.

\section{Contacto:}

Elisabeth, Ramos-Rodríguez, Pontificia Universidad Católica de Valparaíso, Blanco Viel 2950, Valparaíso, Chile, elisabeth.ramos@pucv.cl, (056)322274074.

Pamela Reyes-Santander, Pontificia Universidad Católica de Valparaíso, Blanco Viel 2950, Valparaíso, Chile, pamela.reyes@ucv.cl, (056)322274074. 


\title{
Encouraging reflection of the teacher: a Study of classes on integral calculus using technology
}

\begin{abstract}
To address the question 'how to strengthen a collective environment, reflection on their practice of university professors?' we have designed an alternative education based on a study session Classes articulated with the notion of systematic reflection. We established theoretical framework from the reflection and the notion of mathematical task to address, with a qualitative approach, an interpretive study. We examined data from transcripts of the video recording of the session in an international event. It is noted that alternative training proposal has elements that favor reflection on teaching practice.
\end{abstract}

\section{Key words}

Teacher reflection; study classes; integral calculus; technology.

\section{Introducción}

Los nuevos enfoques educativos, las diversas modalidades de evaluación docente, el cambio generacional entre docentes y estudiantes, los avances tecnológicos, son algunos de los factores que hacen que el desarrollo profesional se deba tomar con seriedad y profundidad en el ámbito educativo. Alsina (2012) detecta tres factores que favorecen el proceso de transformación de las concepciones del profesorado, en donde el segundo de ellos es la reflexión sistemática sobre la propia práctica. Se hace necesario proyectar la reflexión con seriedad y profundidad para lograr un proceso de transformación en las concepciones de los participantes.

Desde esta perspectiva, contar con docentes con habilidades de reflexión y pensamiento crítico, empieza a ser un foco de interés de las políticas educativas a nivel mundial. En Chile se evidencia un aumento de las investigaciones en esta línea, a través del trabajo de Cornejo y Fuentealba (2008) y del trabajo doctoral de Ramos (2014), en México, el estudio de Parada, Figueras y Pluvinage (2009), en el Reino Unido, la investigación de Jaworski (1993), y en Alemania, el trabajo de Reyes-Santander y Ramos-Rodríguez (2015). Un trabajo doctoral que aborda la reflexión de docentes universitarios es el de Briceño (2015), en la que aplica estrategias formativas basándose en la reflexión. En este contexto, surge nuestra pregunta ¿cómo fortalecer en un entorno colectivo generado dentro de un evento internacional, la reflexión sobre su práctica del docente universitario de matemáticas? y se desprende como objetivo de trabajo, diseñar y estudiar una alternativa para favorecer la reflexión de académicos de enseñanza superior, basada en la metodología del Estudio de Clases y la reflexión sistemática.

La conexión del Estudio de Clases y la reflexión docente, no es simple casualidad. Elliott (2010), Uno de los principales representantes de la metodología de investigación-acción en educación, enfatiza el rol relevante que tienen los procesos de reflexión cooperativa como componente clave de la profesionalidad autónoma de los docentes, afirmando que el Estudio de clases proporciona un contexto y un espacio en el que profesores reflexionar sobre la práctica en su aula. En educación matemática, el Estudio de Clases es una de las formas de articular la reflexión compartida con la práctica, teniendo diferentes 
concreciones a nivel mundial (Callejo, Llenares y Valls, 2007), ver el estudio que presentan Lim, White y Chiew (2005).

Los elementos teóricos tienen sus cimientos en la formación de profesores, en particular, en la línea de desarrollo profesional (Flores, 2007; Ponte y Chapman, 2008) y en ella, sobre la reflexión del profesor (Korthagen, Kessels, Koster, Lagerwerf y Wubbels, 2001; Schön, 1983).

El estudio de clase se desarrolló en el marco del Segundo Simposio Latinoamericano para la Integración de la Tecnología en el Aula de Matemáticas y Ciencias, a realizarse en México en el año 2010, se reunieron, un grupo de académicos de la Pontificia Universidad Católica de Valparaíso (Chile), para presentar una propuesta de trabajo colectivo para profesores universitarios, que apunte a la mejora de la práctica pedagógica, a través de la discusión y reflexión de una clase (diseñada y realizada en una universidad chilena). Este trabajo recoge esta propuesta de innovación, la que busca aprovechar las instancias de eventos internacionales, para enfrentar la formación del profesorado, desde la mirada de la reflexión docente.

Con una metodología cualitativa, analizamos la sesión, a través del análisis de contenido (Flick, 2004), de los registros de las grabaciones de vídeo. Del análisis realizado se extraen diversos resultados, de índole disciplinaria y didáctica, que dan luces de cómo enfrentar la formación docente, bajo el alero de la reflexión sobre la práctica.

\section{Marco de referencia y antecedentes}

La formación de profesores es una línea de investigación, ampliamente desarrollada, dentro de la didáctica de las matemáticas, teniendo una diversidad de estudios en esta área, como lo dejan de manifiesto Cardeñoso, Flores y Azcárate (2001), a través de distintas investigaciones que van en esta línea, indicando el sentido que tiene en la docencia de matemáticas. Dentro de esta línea está el estudio del desarrollo profesional, proceso en donde el profesor asume el papel de protagonista de un proceso, iniciado en la formación inicial de la carrera profesional y que evoluciona a lo largo de la vida, tomando en cuenta los sucesos personales como factores que influyen en su desarrollo (Ponte y Chapman, 2008).

La reflexión es un elemento del desarrollo profesional, que se le presta atención en el ámbito docente desde hace varias décadas, cuando Dewey (1910) presentó algunas directrices respecto a la noción de reflexión, manteniéndose presente en la concepción de profesor reflexivo actual (Flores, 2007), que considera, además, la epistemología en la práctica, aportada por Schön (1983), quien relaciona la reflexión con la acción, a través de las nociones de reflexión durante y sobre la acción (ibídem, 1983).

Existen diversos modelos, que permiten abordar la reflexión de manera sistemática, de aquellos utilizados en educación matemática, seleccionamos el modelo ALaCT de Korthagen et al. (2001), empleado en formación inicial y continua del profesorado.

Korthagen (Korthagen et al., 2001), describe el proceso destinado al aprendizaje reflexivo, denominándolo ALaCT, como un proceso cíclico con cinco fases. En la tabla 1, se especifica cada fase, incluyendo algunas preguntas orientadoras, para estimular al docente en torno a cada fase (Tigchelaar, Melief, Rijswijk y Korthagen, 2005). 
Tabla 1.

Fases del proceso ALaCT (Korthagen et al., 2001; Tigchelaar et al., 2005)

\begin{tabular}{|c|c|c|}
\hline Fase & Definición & Preguntas de apoyo \\
\hline $\begin{array}{l}\text { Fase } 1 \\
\text { Acción } \\
\text { experiencia. }\end{array}$ & $\begin{array}{l}\text { Es la situación que da el punto de partida } \\
\text { a la reflexión, de donde se extrae la } \\
\text { problemática que el observador (u } \\
\text { observadores, activo(s) en la clase o no) } \\
\text { identificará y analizará. En esta fase, se } \\
\text { describe lo que pretende la actividad, en } \\
\text { qué se quiere centrar el docente en } \\
\text { forma especial, entre otros. Esta fase } \\
\text { está relacionada con la idea de Dewey de } \\
\text { partir de un problema, una situación } \\
\text { conflictiva o importante para el profesor. }\end{array}$ & $\begin{array}{l}\text { ¿Qué quería conseguir? } \\
\text { ¿En qué me quería centrar de } \\
\text { forma especial? } \\
\text { ¿Qué quería intentar poner en } \\
\text { práctica? }\end{array}$ \\
\hline $\begin{array}{l}\text { Fase } 2 \\
\text { Mirar hacia atrás } \\
\text { en la acción. }\end{array}$ & $\begin{array}{l}\text { Consiste en esbozar una "imagen" de lo } \\
\text { que fue la situación real. }\end{array}$ & $\begin{array}{l}\text { ¿Cuáles fueron los } \\
\text { acontecimientos concretos? } \\
\text { ¿Qué quería? ¿Cómo lo hice? } \\
\text { ¿Cómo me sentí? } \\
\text { ¿Qué, en mi opinión, } \\
\text { pensaban, sentían o hacían mis } \\
\text { alumnos? }\end{array}$ \\
\hline $\begin{array}{l}\text { Fase } 3 \\
\text { Conocimiento de } \\
\text { los puntos } \\
\text { importantes o } \\
\text { esenciales. }\end{array}$ & $\begin{array}{l}\text { Focalizamos la atención en tomar } \\
\text { conciencia de los aspectos } \\
\text { fundamentales que dieron lugar a las } \\
\text { respuestas de la fase anterior. En esta } \\
\text { etapa es donde suele intervenir un } \\
\text { agente externo (en forma de experto o } \\
\text { mediante la lectura de documentos) que } \\
\text { orienten al profesor a examinar las } \\
\text { teorías que subyacen al problema que } \\
\text { ahora se hace explícito. }\end{array}$ & $\begin{array}{l}\text { ¿Cuál es la relación entre las } \\
\text { respuestas dadas a las } \\
\text { preguntas en la fase 2? } \\
\text { ¿Qué influencia tiene el } \\
\text { contexto/la escuela como un } \\
\text { todo? } \\
\text { ¿Qué significa esto para mí? } \\
\text { ¿En qué consiste el problema } \\
\text { (o el descubrimiento positivo)? }\end{array}$ \\
\hline $\begin{array}{l}\text { Fase } 4 \\
\text { Crear, buscar y } \\
\text { preparar } \\
\text { comportamientos } \\
\text { alternativos para } \\
\text { la acción. }\end{array}$ & $\begin{array}{l}\text { En este momento los docentes buscan } \\
\text { estrategias o soluciones de cómo } \\
\text { abordar posteriormente la } \\
\text { problemática(s), para aplicarlas en un } \\
\text { nuevo evento escolar. Se buscan } \\
\text { soluciones o caminos para aprovechar el } \\
\text { descubrimiento de la etapa anterior. }\end{array}$ & $\begin{array}{l}\text { ¿Qué alternativas posibles veo? } \\
\text { (soluciones o caminos para } \\
\text { aprovechar mi } \\
\text { descubrimiento) } \\
\text { ¿Cuáles son las ventajas y } \\
\text { desventajas? } \\
\text { ¿Qué decido hacer la próxima } \\
\text { vez? } \\
\text { ¿Cómo hago eso exactamente } \\
\text { y en concreto? }\end{array}$ \\
\hline $\begin{array}{l}\text { Fase } 5 \\
\text { Comprobar en una } \\
\text { nueva situación. }\end{array}$ & $\begin{array}{l}\text { En esta etapa se estudia una nueva } \\
\text { situación a partir de la reflexión realizada } \\
\text { en el ciclo anterior, empezando un ciclo } \\
\text { nuevo de reflexión, pero desde las } \\
\text { apreciaciones anteriores. }\end{array}$ & \\
\hline
\end{tabular}


Este proceso cíclico, Korthagen lo modela por medio de la figura 1.

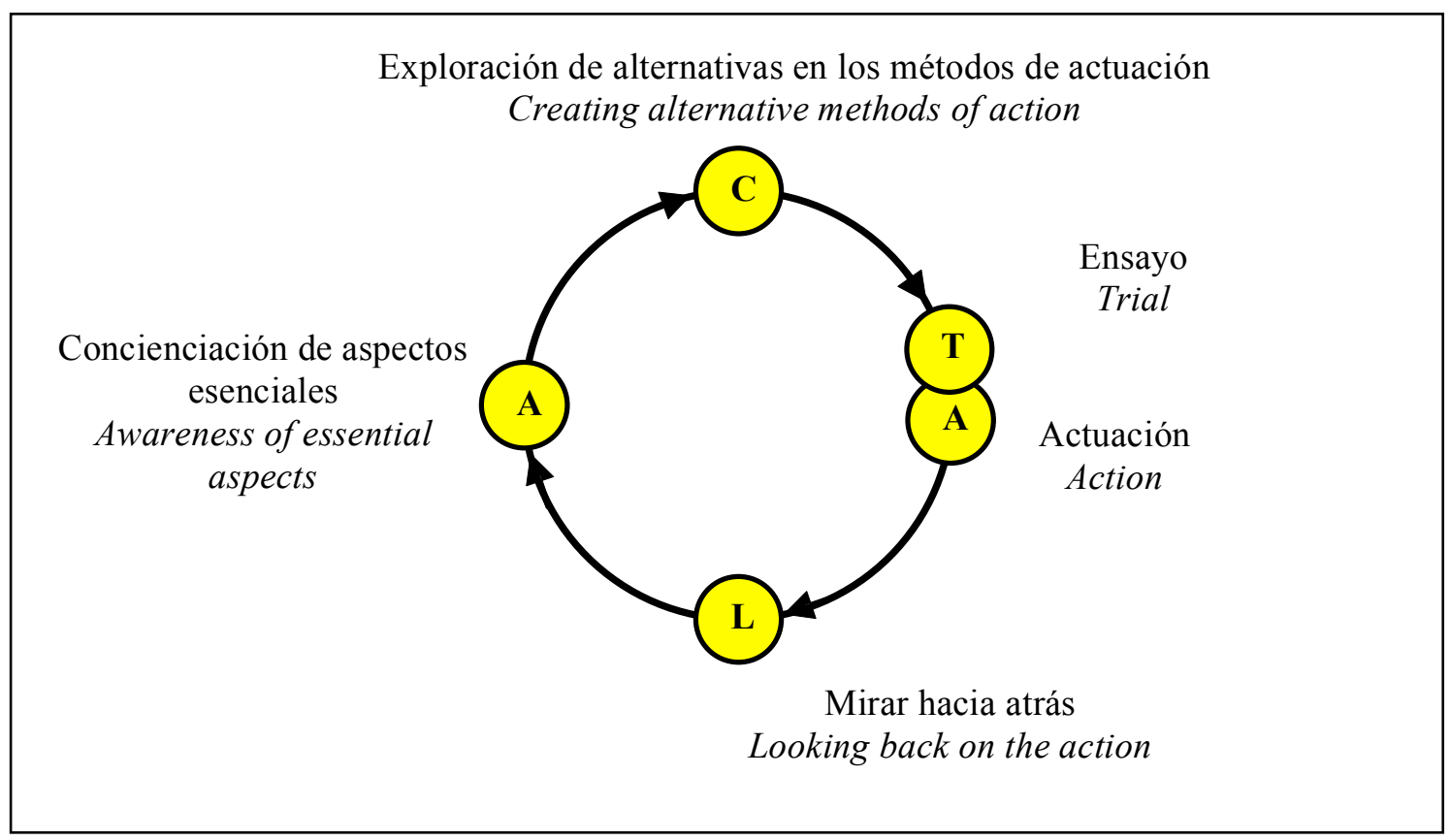

Figura 1. Modelo ALaCT de Korthagen (Korthagen et al, 2001)

La reflexión debe centrarse en un problema de la práctica de los profesores. La sesión analizada, se focalizó en la discusión de problemas que surgen en la enseñanza y el aprendizaje del cálculo integral, en particular, sobre el tratamiento de la noción de integral impropia. Con el propósito de profundizar en aspectos referentes a la problemática discutida, analizamos el elemento matemático que está involucrado en el estudio, desarrollando parte del análisis didáctico (Gómez, 2007; Rojas, Flores y Ramos, 2014) de la integral impropia.

Para analizar la reflexión, es necesario centrarse en algunos elementos que puedan reflejar el proceso llevado a cabo. En este caso, se consideran las tareas de enseñanza discutidas por los docentes y el contenido matemático que emerge.

Sobre las tareas de enseñanza, Stein y sus colegas (Stein, Smith, Henningsen y Silver, 2000), sostienen que la reflexión sobre la enseñanza a través del lente del marco de las tareas matemáticas, proporciona a los profesores la oportunidad de examinar críticamente la actividad del aula, en términos de las exigencias cognitivas que se evidencian en las tareas, cómo se pueden realizar de forma diferente y/o mejorar aspectos de éstas. Destacamos dos aspectos sobre las tareas de enseñanza: gestión y nivel de exigencia cognitiva.

La gestión de las tareas, está relacionada con la forma en que se implementan las tareas en clases. El análisis cognitivo dentro del análisis didáctico (Gómez, 2007; Rico, 1997), permite detallar fundamentadamente: aquellos errores de los estudiantes al abordar las tareas, las dificultades que subyacen a esos errores y los obstáculos que necesitan superar para resolver esas dificultades. Estos tres aspectos consideramos como categorías de análisis respecto a la gestión de las tareas.

La demanda cognitiva, se refiere a la profundidad con que se pretende tratar un tema. Para este análisis, se consideró cuatro categorías de demanda cognitiva, excluyentes entre sí: 
memorización, procedimientos sin conexiones, procedimientos con conexiones y "haciendo matemática" (Stein et al., 2000).

Sobre el contenido matemático, nos basamos en parte del análisis de contenido (Gómez, 2007; Rico, 1997), analizando los conceptos y procedimientos que cubren el tema matemático. Abordamos este análisis, a través de un mapa conceptual con los elementos fenomenológicos, su clasificación y registros de representaciones (figura 2).

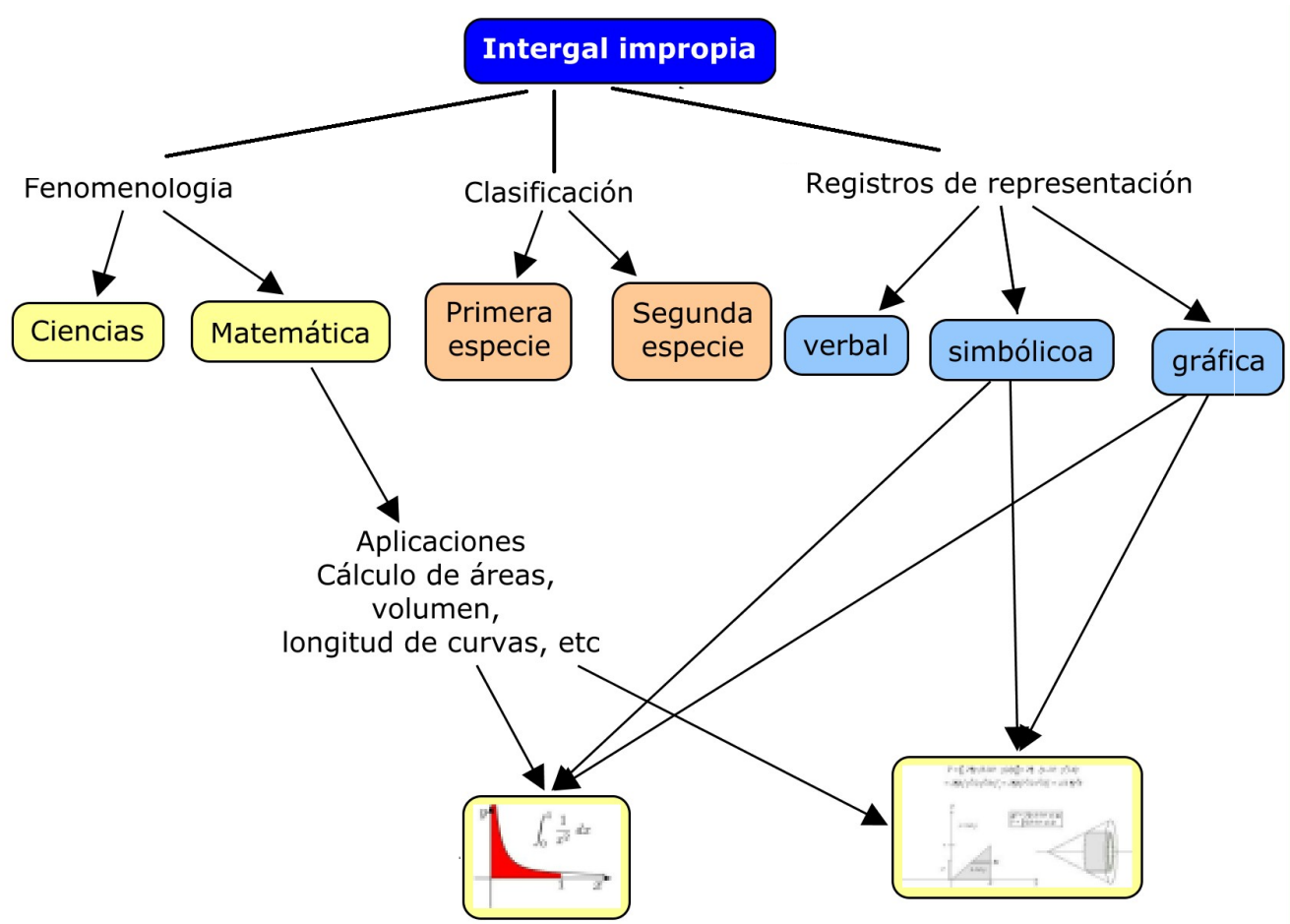

Figura 2. Mapa conceptual con elementos fenomenológicos y representaciones

Y también un mapa conceptual con los objetos matemáticos relacionados con la integral impropia (figura 3). Ambos mapas muestran una panorámica de los elementos involucrados y/o relacionados con las integrales impropias (dentro de los contenidos matemáticos de la enseñanza universitaria), de las formas de representación que utilizan y de los campos de fenómenos que la rodean. 


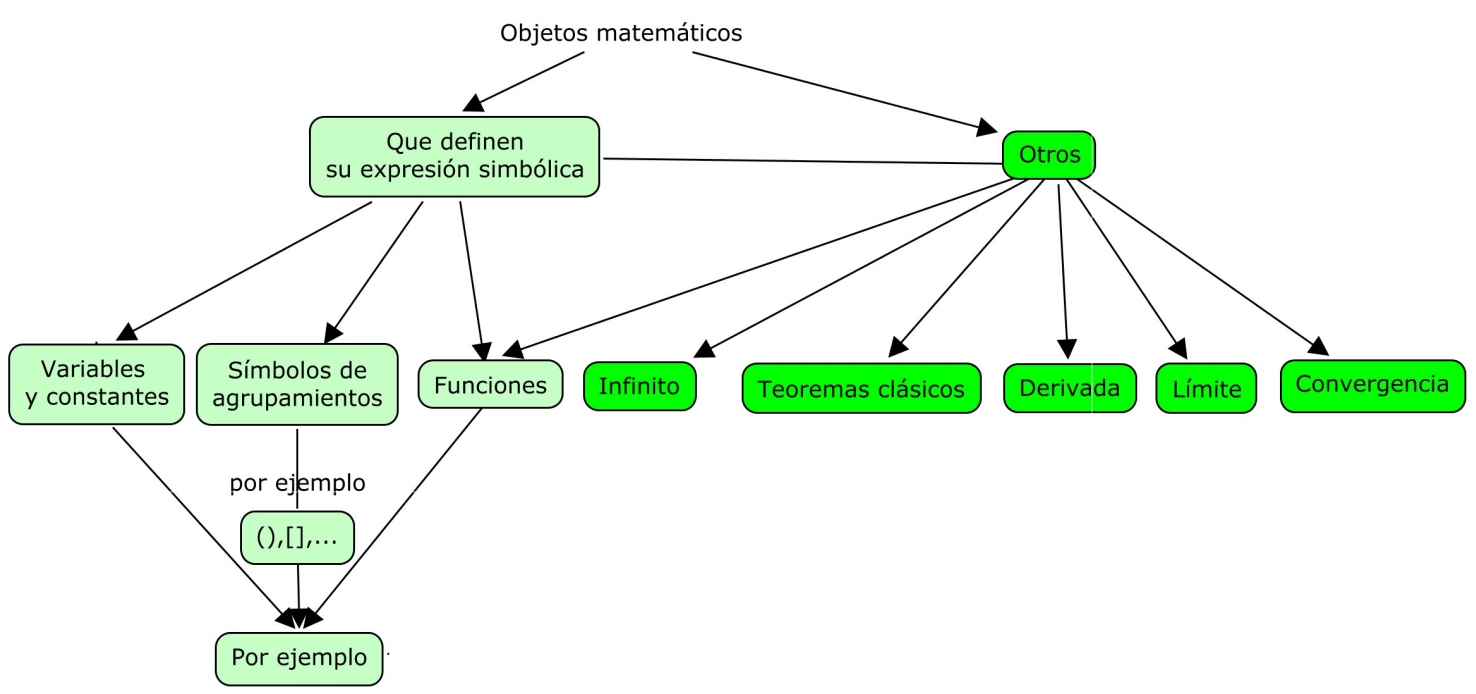

Figura 3. Mapa conceptual con objetos matemáticos relacionados con integral impropia

Esta figura muestra las consideraciones que pueden surgir en torno a la integral en los diferentes etapas de reflexión, dependiendo de la profundización los profesionales de la educación, estos pueden ir cada vez introduciéndose más en los diferentes aspectos de la integral.

Hemos visto elementos teóricos y antecedentes respecto a la reflexión, pasamos ahora a mostrar algunos antecedentes que colaboran al estudio.

El uso de medios tecnológicos en la enseñanza universitaria, va tomando mayor fuerza como un elemento favorecedor para el aprendizaje de los estudiantes (Baelo y Cantón, 2009). Aun así, se percibe cierta resistencia, por parte del profesorado, al cambio pedagógico que su uso involucra (Lerman y Zevenbergen, 2006). En contraparte, aumentan cursos e instancias de formación para docentes sobre TIC. En eventos internacionales, destacamos los Simposios Latinoamericanos para la Integración de la Tecnología en el Aula de Matemáticas y Ciencias (http://education.ti.com/educationportal/sites/LATINOAMERICA/nonProductMulti/simposio. html?bid=3), dirigido a especialistas de la educación, con el fin de capacitar en tecnologías específicas y favorecer el encuentro de docentes para compartir experiencias. En él, resaltamos una instancia que involucra la realización de clases en situ, con tecnología, con objeto de analizarlas con el profesorado presente en el evento. De estas instancias surge la experiencia que analizamos en este documento, la que posteriormente, replicamos con académicos de la Universidad de Ausburgo (Ramos, 2012).

Una experiencia similar, con tecnología, pero enmarcada dentro de un curso de formación continua, realizamos con docentes de enseñanza primaria, secundaria y universitaria (Ramos y González, 2012), en el contexto de Estudio de Clases japonés (Isoda, Mena y Arcavi, 2007).

Se han diseñado otras alternativas de trabajo con Estudio de Clases, como Nickerson, Fredenberg y Druken (2014), donde diseñan y llevan a cabo una estrategia de Estudio de Clases on-line.

Teniendo sentado nuestro marco teórico y considerando los estudios relacionados con la temática, pretendemos estudiar una alternativa de formación -para profesores de enseñanza superior- basada en el Estudio de Clases y la reflexión, en un encuentro 
internacional de profesores sobre uso de tecnologías, planteándonos dos objetivos específicos: a) explicitar la conexión entre la sesión de clase (Estudio de Clases) con el proceso de reflexión, es decir, identificar los momentos de la sesión de Estudio de Clases con las fases del proceso reflexivo promovido, b) caracterizar los elementos predominantes en la reflexión llevada a cabo por los docentes.

\section{Método}

Con un enfoque cualitativo, realizamos un estudio exploratorio, cuyo diseño es no experimental, transversal (Hernández, Fernández y Baptista, 2010). El contexto es una sesión de Estudio de Clases realizada en el año 2010, dirigido por dos profesoresinvestigadores de la Pontificia Universidad Católica de Valparaíso, que se propusieron promover instancias de desarrollo profesional para docentes de matemáticas a través del diseño, realización y discusión de una clase que utilice tecnología, que llamamos Clase en Estudio y que detallamos a continuación.

\section{La Clase en estudio}

Es una clase diseñada con uso de tecnología inalámbrica (Calculadoras TI Nspire conectadas a TI Navigator), con el fin de introducir el concepto de integral impropia. La tarea de enseñanza prevista consta de cuatro partes, como se muestra en la figura 4.

Parte I: Lectura y comprensión del enunciado.

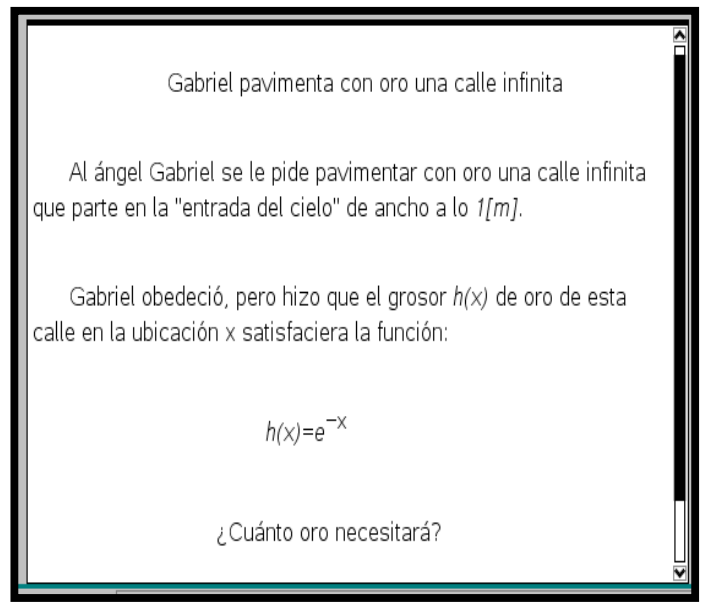

Parte III: Uso de parámetros, deslizadores

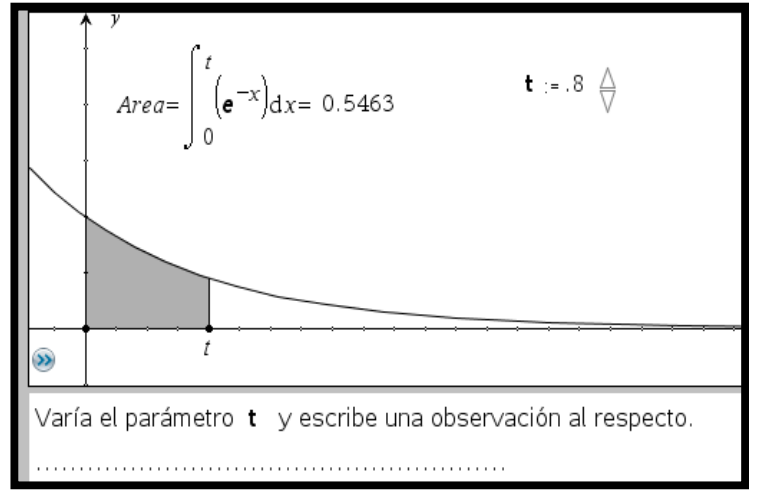

Parte II: Uso de registro de representación.

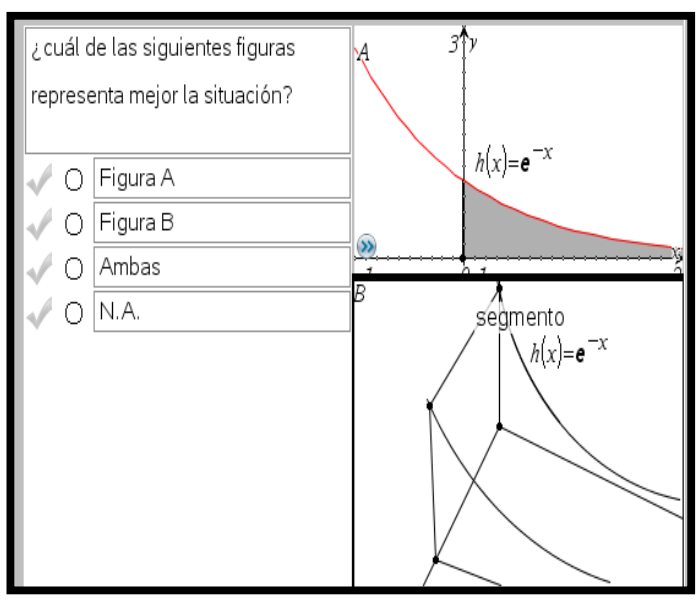

Parte IV: Concluyendo.

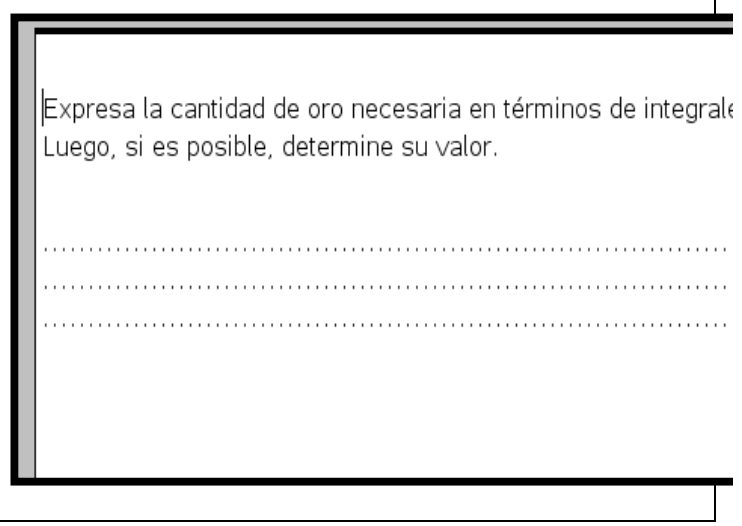

Figura 4. Las cuatro partes que componen la tarea de enseñanza 
La clase fue analizada en la sesión de Estudio de Clases, describiéndola a continuación.

La sesión de Estudio de Clases

Se basó en las ideas que emergen del Estudio de Clases, tuvo cinco momentos claves, los que se resumen en la tabla 2.

Tabla 2.

Momentos de la sesión de Estudio de Clases

\begin{tabular}{|c|c|c|}
\hline Momentos & \multicolumn{2}{|l|}{ Especificaciones } \\
\hline \multirow{7}{*}{$\begin{array}{l}\text { 1. Presentación de } \\
\text { aspectos de la } \\
\text { clase }\end{array}$} & \multirow{3}{*}{$\begin{array}{l}\text { Objetivos } \\
\text { planteados }\end{array}$} & Ampliar el concepto de integral impropia \\
\hline & & Usar las bondades de TI Nspire \\
\hline & & Usar las bondades de TI Navigator \\
\hline & \multirow{2}{*}{$\begin{array}{l}\text { Contexto de la } \\
\text { clase }\end{array}$} & Características de los alumnos. \\
\hline & & $\begin{array}{l}\text { Conceptos previos: integrales definidas, aplicación de } \\
\text { éstas }\end{array}$ \\
\hline & \multirow{2}{*}{$\begin{array}{l}\text { Recursos } \\
\text { tecnológicos }\end{array}$} & TI Nspire \\
\hline & & TI Navigator \\
\hline $\begin{array}{l}\text { 2. Presentación } \\
\text { de los } \\
\text { participantes }\end{array}$ & $\begin{array}{l}\text { Breve } \\
\text { presentación de } \\
\text { participantes }\end{array}$ & $\begin{array}{l}\text { Para promover un clima de confianza, se les pide a los } \\
\text { docentes que se presenten, detallando su lugar de } \\
\text { trabajo, cursos y/o alumnos a los que dicta clases. } \\
\text { También se les pide que manifiesten si han tenido } \\
\text { experiencia en uso de TIC y a qué nivel. }\end{array}$ \\
\hline \multirow{4}{*}{$\begin{array}{l}\text { 3. Presentación } \\
\text { de la clase }\end{array}$} & \multirow{4}{*}{$\begin{array}{l}\text { De las partes de la } \\
\text { clase. Análisis a } \\
\text { priori }\end{array}$} & Parte I: Lectura y comprensión del enunciado \\
\hline & & $\begin{array}{l}\text { Parte II: Uso de registros de representación, cambio } \\
\text { de dimensiones }\end{array}$ \\
\hline & & Parte III: Uso de parámetros, deslizadores \\
\hline & & Parte IV: Concluyendo \\
\hline \multirow{2}{*}{$\begin{array}{l}\text { 4. Presentación } \\
\text { en vídeo de la } \\
\text { clase }\end{array}$} & \multirow{2}{*}{$\begin{array}{l}\text { De algunos } \\
\text { episodios de cada } \\
\text { parte de la clase }\end{array}$} & Presentación de episodios \\
\hline & & $\begin{array}{l}\text { Confrontación con las respuestas y análisis a priori } \\
\text { realizado anteriormente }\end{array}$ \\
\hline 5. Metareflexión & $\begin{array}{l}\text { Planteamiento de } \\
\text { preguntas } \\
\text { globales }\end{array}$ & $\begin{array}{l}\text { ¿Se desarrolló la clase según lo que se pensó? } \\
\text { ¿Cómo influye la concepción de "infinito" para el } \\
\text { desarrollo de la actividad? } \\
\text { ¿Por qué, a pesar de haber realizado un análisis } \\
\text { gráfico, los alumnos no relacionan éste análisis con la } \\
\text { convergencia de la integral impropia? } \\
\text { ¿Qué aspectos habría que reconsiderar para una } \\
\text { próxima oportunidad? } \\
\text { ¿Realizaría esta tarea con sus alumnos? }\end{array}$ \\
\hline
\end{tabular}


Participan 18 docentes de matemáticas, de 11 universidades de México y de una universidad chilena. Fue dirigida por dos profesores-investigadores, uno de ellos es la autora principal de este artículo.

El instrumento de recogida de información se basa en las transcripciones de la grabación en video de la sesión de Estudio de Clases, comentarios, ideas y observaciones de los profesores en dichas transcripciones, textos escritos de las intervenciones de los profesores.

Para extraer la información, realizamos un análisis de contenido (Krippendorff, 1990), fijando como unidades de análisis el conjunto de intervenciones de los participantes que tuvieran alguna conexión o idea en común, las que llamamos "episodios".

Las dimensiones, de índole cualitativas, que nos permitieron clarificar el proceso de reflexión realizado por los docentes son las tareas de enseñanza y el contenido matemático. Las categorías de análisis empleadas para el análisis de contenido, respecto a la dimensión tareas de enseñanza, son la forma de gestionar las tareas y el nivel de exigencia cognitiva que proponen. Para la dimensión contenido matemático, las categorías se extraen del análisis de contenido (del análisis didáctico) desarrollado: concepto, registros de representación, clasificación y aplicaciones. Los detalles sobre las categorías fueron expuestas en el marco teórico. La tabla 3, recoge las dimensiones y categorías de manera resumida.

Tabla 3.

Dimensiones y categorías de análisis

\begin{tabular}{|l|l|l|}
\hline Dimensión & Categorías & Sub-categorías \\
\hline $\begin{array}{l}\text { Tareas de } \\
\text { enseñanza }\end{array}$ & Gestión de tareas & $\begin{array}{l}\text { Errores } \\
\text { Dificultades } \\
\text { Obstáculos }\end{array}$ \\
\cline { 2 - 3 } $\begin{array}{l}\text { Contenido } \\
\text { matemático }\end{array}$ & Comanda cognitiva & $\begin{array}{l}\text { Memorización } \\
\text { Producción sin conexión } \\
\text { Producción con conexión } \\
\text { Haciendo matemáticas }\end{array}$ \\
\cline { 2 - 3 } & $\begin{array}{l}\text { Registros } \\
\text { representación }\end{array}$ & $\begin{array}{l}\text { Noción } \\
\text { Objetos matemáticos con los que se } \\
\text { relaciona }\end{array}$ \\
\cline { 2 - 3 } & Clasificación & $\begin{array}{l}\text { Verbal } \\
\text { Simbólico } \\
\text { Gráfico }\end{array}$ \\
\cline { 2 - 3 } & Fenomenología & $\begin{array}{l}\text { De primera especie } \\
\text { De segunda especie }\end{array}$ \\
\cline { 2 - 3 } & $\begin{array}{l}\text { Contextos físicos } \\
\text { Contextos matemáticos } \\
\text { Otros }\end{array}$ \\
\hline
\end{tabular}


Teniendo definidas las categorías de análisis, procedimos a examinar las intervenciones de los profesores y clasificar sus aportes. Para ello, identificamos las categorías sobre cada unidad de análisis extraída de las transcripciones.

En el caso que la unidad de análisis corresponda a la intervención de un docente, hemos asignado la sigla I(D). Cuando es uno de los dos profesores-investigadores la sigla es I(P1) o $\mathrm{I}(\mathrm{P} 2)$, según sea el que intervenga de uno de los dos.

\section{Análisis y discusión}

Antes de analizar la reflexión de los docentes, se procede a articular los momentos de la sesión de Estudio de Clases con las fases del ciclo reflexivo ALaCT, proceso que detallaremos a continuación.

La primera fase del proceso reflexivo se inicia con una situación que emerge de los profesores-investigadores, quienes presentan una clase (momento 1 de la sesión de Estudio de Clases), y provocan a los docentes la discusión sobre lo que podría suceder en ella (momento 3). También es parte de esta fase, el momento 2, donde los profesoresinvestigadores provocan un clima de confianza entre los participantes, colaborando al proceso de reflexión de ellos. El momento 4 se identifica con las fases 2 y 3 del ciclo reflexivo, ya que en él, los docentes, esbozan lo que sucedió e identifican elementos importantes de ello, a través del apoyo de expertos (en este caso, los profesoresinvestigadores). La fase 4, surge cuando los docentes nuevamente analizan la clase, pero en su totalidad, sin fijar la atención en las partes de ésta, buscando globalmente elementos que puedan servir para mejorarla, reformulando aspectos de ésta (momento 5). Debido a la dificultad de contar con un nuevo grupo de alumnos, bajo condiciones similares a las que tenían los alumnos de la clase presentada, no se pudo concretar la fase 5 del ciclo de reflexión, es decir, llevar a cabo la clase reformulada.

De esta forma, vemos como las cuatro primeras fases del ciclo de reflexión, han sido identificadas dentro de los momentos de la sesión, como se resume en la figura 5 .

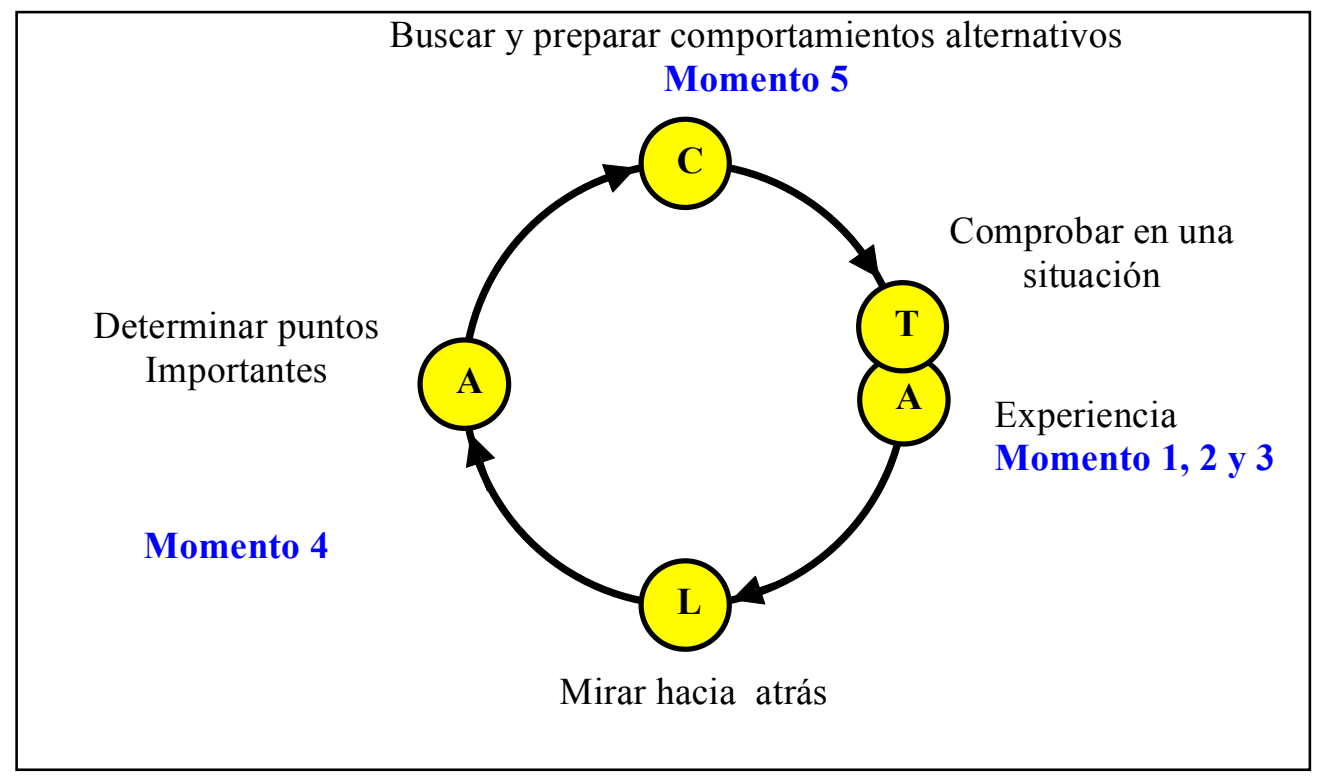

Figura 5. Las fases del modelo ALaCT concretado en el curso formativo 
Luego de articular la sesión de trabajo con las fases del proceso reflexivo, pasamos a identificar y detallar elementos claves en cada fase.

La fase primera, se inicia con una situación planteada por los profesores-investigadores a cargo de la sesión de Estudio de Clases. Esta situación consistió en el planteamiento de una clase, su diseño y discusión (a priori) sobre los aspectos relevantes de la clase (momento 1 y 3 de la sesión de Estudio de Clases).

En esta fase, los profesores participantes se familiarizan con elementos generales de la clase (momento 1), como son los objetivos de ésta, los recursos utilizados y algunas características de los alumnos, como, por ejemplo, sus conocimientos previos y la profesión que estudian. También los docentes en esta fase, se familiarizan con las partes de la clase (momento 3), a través de la actividad diseñada con la calculadora TI Nspire y TI Navigator. En este tercer momento, además los docentes discuten (a priori) sobre lo que creen que pueda suceder en cada parte de la clase, planteándoles preguntas del tipo: ¿qué crees que va a responder el alumno? ¿Qué conclusiones obtendrá el alumno cuando varíe el parámetro $t$ en la calculadora? Estas y otras preguntas son cruciales para avanzar en el proceso de reflexión, ya que ellas, por una parte, permiten a los docentes extraer sus creencias sobre las dificultades y errores que puedan surgir en la clase respecto a la matemática misma, al uso de tecnología, o a otro aspecto de ésta. Las ideas generales de esta discusión se dejan en la pizarra de tal forma de poder contrastarlas posteriormente en la fase siguiente, como se muestra en la figura 6.

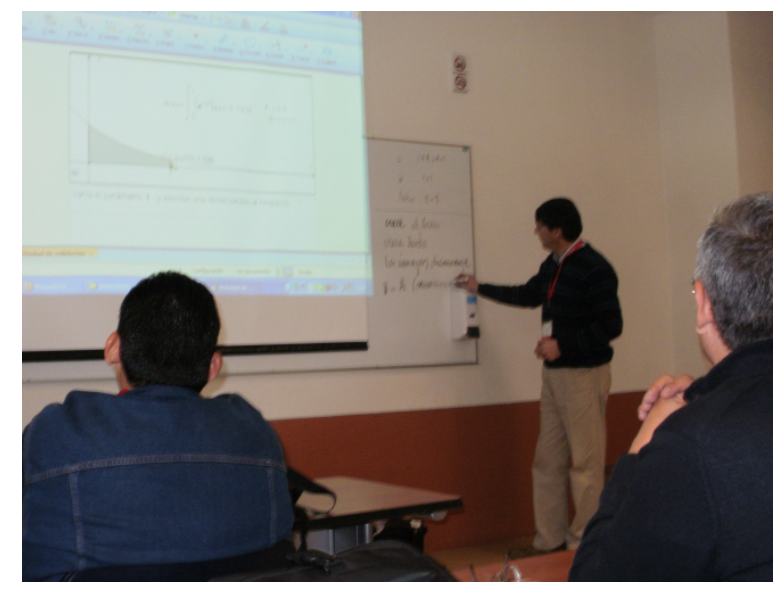

Figura 6. Evidencia del trabajo en la pizarra

Un ejemplo destacable de las opiniones vertidas por los docentes en esta fase, es el episodio de la tabla 4, donde se percibe una seguridad en los docentes, respecto a que los estudiantes no tendrán dificultad (categoría gestión) en deducir la relación entre el parámetro asociado a la integral y la convergencia involucrada, argumentando que los conceptos previos, relativos a la noción de límite y convergencia (categoría concepto), están adquiridos por los estudiantes. Esto queda en evidencia en el episodio presentado en la tabla 4. 
Tabla 4.

Trascripción del episodio 34

\begin{tabular}{|l|l|}
\hline I(D) & $\begin{array}{l}\text { El alumno va a observar el número al cual topa, porque a veces se mueven } \\
\text { mucho al parámetro que uno les da la oportunidad de manipular, y observar que } \\
\text { el resultado es ese o que repiten la operación y vuelve a ser el que fue la primera } \\
\text { vez y deducen que se dirige, que converge, pero si observan que el valor por } \\
\text { hacerlo variar se acerca a tal tope. }\end{array}$ \\
\hline I(D) & $\begin{array}{l}\text { Si lo van a deducir, porque ellos ya vieron el concepto de límite, en el concepto } \\
\text { de límite se ve la convergencia. Entonces, lo más probable es que conforme } \\
\text { muevan el deslizador a la derecha lleguen a la conclusión de que hay una } \\
\text { convergencia, el concepto de límite. }\end{array}$ \\
\hline I(P1) & $\begin{array}{l}\text { Eso es lo que queremos, ese es un alumno ideal, que el concepto de limite lo } \\
\text { traigan acá... }\end{array}$ \\
\hline
\end{tabular}

También hay una preocupación por abordar la gestión de las tareas de enseñanza, por ejemplo, al buscar (en la Parte II de la clase) algunas preguntas que dirijan el aprendizaje de los alumnos o respecto a la formalización del contenido matemático en discusión. En esta preocupación se percibe una tendencia a dirigir los procedimientos de los alumnos, como queda en manifiesto en el episodio siguiente. Los profesores no advierten que este planteamiento provoca una baja en el nivel de exigencia cognitiva (categoría demanda cognitiva) de la tarea de enseñanza, aspecto que luego de este episodio es planteado por uno de los profesores-investigadores.

Tabla 5.

Transcripción del episodio 38

\begin{tabular}{|l|l|}
\hline I(D) & $\begin{array}{l}\text { Para eso se requeriría una serie de preguntas guiadas para que el alumno lo } \\
\text { pueda precisamente llevar a lo que uno quiere que vaya. }\end{array}$ \\
\hline I(D) & $\begin{array}{l}\text { A lo mejor también hacer preguntas... ¿Cómo crece el área? ¿A qué término se } \\
\text { acerca? Estas preguntas formularlas para que ellos las deduzcan. }\end{array}$ \\
\hline
\end{tabular}

Una de las intervenciones del momento 2, que también pertenece a esta fase inicial, se presenta en la tabla 6 . En ella un docente manifiesta su experiencia en el uso de tecnologías en el aula, refiriéndose a aspectos de la gestión de las tareas de enseñanza (forma de llevarlas a cabo y el rol de recursos tecnológicos usados). También se refiere a aspectos sobre los contenidos matemáticos, en particular, si los estudiantes tienen un dominio procedimental o bien conceptual sobre algunos contenidos. 
Tabla 6.

Transcripción del episodio 45

I(D) Realmente estoy interesado en esto, o sea, de alguna manera tienes que llevar al "chavito" (o al alumno), a la parte de la pizarra y explicarle con números, y bueno, lo que haces me parece bastante ideal, ya lo llevas (al alumno) a un punto en la pizarra, luego lo llevas a una situación de necesidad, por así decirlo) y luego te lo llevas a la parte donde ya está mecanizado, donde ya está más familiarizado y puede interactuar y jugar más con el equipo. Porque en algunos casos, lo he visto, tienes algunos jóvenes, muchachos, que agarran la máquina (calculadora) y si lo saben (creen saberlo) y ahí está todo el rollo, pero de pronto le dices ¿qué sucede aquí? y queda todo sorprendido...

En la segunda fase, se intenta lograr que los docentes esbocen cuáles son los acontecimientos que realmente sucedieron. El momento 4 de la sesión es clave para esta fase, la que se mezcla con la fase tres, donde los docentes toman conciencia de los aspectos fundamentales que dieron lugar a las respuestas dadas por ellos en la fase 3. La intervención de agentes externos en la fase 4, en forma de profesores-investigadores, es relevante para orientar a los profesores a examinar las teorías que subyacen a la situación que ahora se hace explícito. Las tablas 7 y 8 , muestra intervenciones de los docentes en la fase 2 y la fase 3 respectivamente.

Tabla 7.

Transcripción del episodio 51

\begin{tabular}{|l|l|}
\hline I(D) & $\begin{array}{l}11 \text { alumnos respondieron a, } 12 \text { alumnos respondieron b y solo } 3 \text { alumnos } \\
\text { respondieron ambas. }\end{array}$ \\
\hline I(D) & $\begin{array}{l}\text { Y aquí al revés (apuntando las respuestas esperadas por ellos en el análisis a } \\
\text { priori)... (risas). }\end{array}$
\end{tabular}

En este episodio los docentes confrontan las respuestas dadas por los estudiantes en la Parte II con las respuestas que ellos esperaban (y discutieron en el análisis a priori). Luego, en el episodio siguiente, luego del aporte de uno de los profesores-investigadores, se observa una toma de conciencia sobre lo que ocurrió realmente y porqué sucedido así. Se percibe un interés por entender a sus estudiantes para poder adelantarse y decir a priori sus razonamientos (errores y/o dificultades en este caso, categoría gestión). 
Tabla 8.

Transcripción del episodio 52

\begin{tabular}{|l|l|}
\hline I(P2) & Pero eso es lo que pensamos que ocurriría. \\
\hline I(D) & $\begin{array}{l}\text { O sea que no comprendemos el razonamiento de los alumnos... Nos hace falta } \\
\text { tratar de entender como el alumno piensa... }\end{array}$ \\
\hline
\end{tabular}

Sobre errores que puedan cometer los alumnos en la realización de la tarea, los docentes observan que la tarea de enseñanza plateada de esa forma puede sugerir un error relacionado con la confusión entre área y volumen. La tabla 9 es un ejemplo de esto.

Tabla 9.

Transcripción del episodio 73

I(D) Una observación que veo ahí, es que va implícita la propiedad de neutro multiplicativo. Al decir que tiene un metro de ancho, a veces el alumno puede estar confundiendo el área con el volumen, porque la respuesta es la misma, tanto el área como el volumen, numéricamente es la misma respuesta. Entonces ¿no han probado con un ancho diferente de 1?, para tratar de que no vayan a tener alumnos que estén con este error que se genera. Mi trabajo ahora está abocado al estudio del error que comete el alumno, el contexto algebraico, al final de cuentas es una implicación.

El contenido matemático también se discute en esta fase, especialmente en relación a la convergencia, al límite de sucesiones y al concepto de infinito.

El obstáculo (categoría gestión) que tuvieron los alumnos con la noción del infinito (categoría concepto y objetos matemáticos relacionados) se discute fuertemente, donde se observa como la mayor parte de los estudiantes no acepta la posibilidad de tener una superficie infinita con un volumen finito.

De esta forma, en la fase cuatro, los docentes, están preparados para buscar estrategias o soluciones de cómo abordar posteriormente una clase con las condiciones similares a la presentada y estudiada. Esto se consolida en el momento 5 . La tabla 10, ilustra una de las intervenciones de los docentes en esta fase, en donde, se buscan estrategias que ayuden a afrontar la tarea de enseñanza de mejor forma por los estudiantes. Así este docente, luego de plantear la posibilidad que el estudiante al iniciar la temática de la integral impropia considere a la integral definida como un todo, como un objeto (olvidando su definición en base a sumas de Riemann), sugiere que retome su definición, vuelva a discretizar (sumas de Riemann), de tal forma que esto le ayude a abordar con mayor facilidad la nueva noción que se presenta (integral impropia). 
Tabla 10.

Transcripción del episodio 98

$\mathrm{I}(\mathrm{D})$

Hay otra posibilidad que el objeto, o sea, que la integral definida sea convertida en un objeto para el estudiante [con intención de utilizarla en la integral impropia], pero al tratar de escudriñar esta situación [referida a la integral impropia], creo que tiene que regresar, tiene que analizar lo que es una integral definida [no mirarla como objeto]. Creo que una manera de ponerlo [la tarea de enseñanza] más alcance del estudiante podría ser considerar el discretizar un poco el problema, es decir, pensar en las " $n$ " en lugar de las " $t$ ", para acercarlo [al estudiante] a la posibilidad de pensar esto como una sucesión. Me parece que es una alternativa...

En este episodio también se puede observar un nuevo elemento matemático que aporta a la discusión, la Suma de Riemann (categoría contenido matemático).

La fase cinco, por las circunstancias del evento, no se pudo llevar a cabo.

De esta forma, se observa como cuatro de las cinco fases el ciclo de reflexión se fueron cubriendo e incentivando en la sesión de Estudio de Clases, con el fin de ser aprovechadas por el grupo de docentes.

\section{Conclusiones}

A la luz de los resultados obtenidos del análisis descrito, se observa que los docentes participantes experimentan una sesión donde se ha incentivado un proceso de reflexión para ellos. En este proceso distinguimos aspectos de las tareas de enseñanza y del contenido matemático discutido. De esta forma, el análisis nos permite percibir cómo los profesores manifiestan preocupación por elementos relativos a las tareas de enseñanza, principalmente relacionados con los errores y las dificultades de los alumnos en el tratamiento de la integral impropia, es decir, elementos de la gestión de las tareas de enseñanza. Aun así, se observa poca alusión por parte de los profesores sobre el nivel de exigencia cognitiva de las tareas de enseñanza. Por otro lado, respecto a la dimensión contenido matemático, vemos que a medida que se avanza en las fases del proceso reflexivo, las intervenciones de los profesores van incorporando nuevos objetos matemáticos, relacionados con la tarea propuesta, lo que enriquece la discusión, aportando con nuevas aristas al problema planteado.

Hemos identificamos elementos de las distintas fases del ciclo reflexivo que se manifestaban en las intervenciones de los distintos participantes de esta experiencia. Destacamos la instancia reflexiva que provocó esta sesión del evento para fomentar la reflexión en la práctica, de profesores de distintas latitudes. En donde, se evidenció un esfuerzo e interés (consciente o no) de los profesores, por desarrollar y exteriorizar aspectos que involucran reflexión en ellos. Se constata como en la sesión de Estudio de Clases fue posible promover cuatro de las cinco fases del proceso de reflexión ALaCT, manifestado en la articulación de la sesión con las fases del ciclo reflexivo.

Por último, es menester mencionar que, a raíz de esta experiencia, se consolidó un grupo de docentes e investigadores chilenos que amplió la sesión a un evento nacional para profesores. Esto se concreta en el "Simposio de Estudio de Clases de Matemática con Tecnología” en el año 2012, y posteriormente su segunda versión en el año 2013 
extendiéndolo para material manipulativo educativo. En ambas versiones participaron alrededor de 50 docentes, donde pretendió materializar las etapas previas a la reflexión sobre la práctica, es decir, la reflexión para y en la práctica, en el que los mismos profesores participantes puedan diseñar y presenciar la clase.

De esta forma, sostenemos que la experiencia fomentó la reflexión del docente universitario, intentando fortalecer un aspecto de su desarrollo profesional, tomando relevancia y pertinencia, dentro de los estudios de la línea de investigación de formación de profesores y dentro de eventos a nivel nacional e internacional.

\section{Referencias}

Alsina, A. (2012). Proceso de transformación de las concepciones del profesorado sobre la resolución de problemas matemáticos. Enseñanza de las ciencias: revista de investigación y experiencias didácticas, 30(3), 71-88. doi: 10.5565/rev/ec/v30n3.645

Arcavi, A. (2003). The role of visualisation representations in the learning of mathematics. Educational Studies in Mathematics, 52, 215-241. doi:10.1023/A:1024312321077

Baelo, R, y Cantón, I. (2009). Las tecnologías de la información y la comunicación en la Educación Superior. Estudio descriptivo y de revisión. Revista Iberoamericana de Educación, 50(7), Recuperado de https://dialnet.unirioja.es/servlet/articulo?codigo=3069990.

Briceño, M. J. (2015). La argumentación y la reflexión en los procesos de mejora de los profesores universitarios colombianos de ciencia en activo: Aplicación de estrategias formativas sobre ciencia, aprendizaje y enseñanza. Enseñanza de las ciencias: revista de investigación y experiencias didácticas, 33(2), 253-254. doi: http://dx.doi.org/10.5565/rev/ensciencias.1590.

Callejo, M. L., Llinares, S., y Valls, J. (2007). El uso de videoclips para una práctica reflexiva. Comunicación presentada en las XIII Jornadas de Aprendizaje y Enseñanza de las Matemáticas - JAEM. Universidad de Granada, Granada, España.

Cardeñoso, J. M., Flores, P., y Azcárate, P. (2001). El desarrollo profesional de los profesores de matemáticas como campo de investigación en educación matemática. En P. Gómez y L. Rico (Eds.) Iniciación a la investigación en didáctica de la matemática. Homenaje al profesor Mauricio Castro (pp. 233-244). Granada: Publicaciones de la Universidad de Granada.

Cornejo, J. y Fuentealba, R. (Ed.) (2008). Prácticas reflexivas para la formación profesional docente: ¿Qué las hace eficaces? Santiago: USACH.

Dewey, J. (1910). How we think. Boston: D.C. Heath.

Flick, U. (2004). Introducción a la investigación cualitativa. Madrid: Morata.

Flores, P. (2007). Profesores de Matemáticas reflexivos: formación y cuestiones de investigación. $\quad$ PNA, 1(4), 139-159. Recuperado de http://www.pna.es/Numeros2/Volumen1Numero4.html.

Gómez, P. (2007). Desarrollo del conocimiento didáctico en un plan de formación inicial de profesores de matemáticas de secundaria. Tesis doctoral en Didáctica de las matemáticas, Universidad de Granada, Granada, España.

Hernández, R., Fernández, C. y Baptista, P. (2010). Metodología de la investigación. México: McGraw-Hill. 
Isoda, M., Arcavi, A. y Mena, A. (2007). El Estudio de Clases Japonés en Matemáticas. Su importancia para el mejoramiento de los aprendizajes en el escenario global. Valparaíso: Ediciones Universitarias de Valparaíso.

Jaworski, B. (1993). The professional development of teachers: The potential of critical reflection. British Journal of In-service Education, 19, 37-42. doi: 10.1080/0305763930190307.

Korthagen, F., Kessels, J., Koster Lagerwerf, B., \& Wubbels, T. (2001). Linking practice and theory: The pedagogy of realistic teacher education. Mahwah, NJ: Lawrence Erlbaum Associates.

Lerman, S., \& Zevenbergen, R. (2006). Maths, ICT and pedagogy: An examination of equitable practice in diverse contexts. In J. Novotná, H. Moraová, M. Krátká, \& N. Stehlíková (Eds.), Proceedings 3oth Conference of the International Group for the Psychology of Mathematics Education, Vol. 4 (pp. 49-56). Prague: PME.

Lim, C.S., White, A., \& Chiew, C.M. (2005). Promoting Mathematics Teacher Collaboration through Lesson Study: What Can We Learn from Two Countries' Experience? Paper presented at the 8th International Conference of The Mathematics Education into the 21st Century Project: "Reform, Revolution and Paradigm Shifts in Mathematics Education" (pp. 135-139), Universiti Teknologi Malaysia (UTM), Malaysia.

Nickerson, D., Fredenberg, M., \& Druken, B. (2014). Hybrid lesson study: extending lesson study on-line, International Journal for Lesson and Learning Studies, 3(2), 152-169. doi: http://dx.doi.org/10.1108/IJLLS-06-2013-0037

Parada, S. E., Figueras, O., y Pluvinage, F. (2009). Hacia un modelo de reflexión de la práctica profesional del profesor de matemáticas. En M. González, M. T. González \& J. Murillo (Eds.), Investigación en Educación Matemática XIII (pp. 355-366). Santander: SEIEM.

Ponte, J.P., \& Chapman, O. (2008). Preservice Mathematics Teachers' Knowledge and development. En L. English (Ed.) Handbook of International Research in Mathematics Education (pp. 225-236). New York, NY: Routledge.

Ramos, E. (2012, enero). Reflexión docente sobre una clase de cálculo integral en un ambiente tecnológico. Conferencia realizada en "Oberseminar zu Didaktik der Mathematik", Universidad de Ausgburg, Alemania.

Ramos-Rodríguez, E. (2014). Reflexión docente sobre la enseñanza del álgebra en un curso de formación continua (Disertación Trabajo Doctoral, Universidad de Granada, Granada). Recuperado

de https://www.researchgate.net/publication/282850359_REFLEXION_DOCENTE_SOBRE _LA_ENSENANZA_DEL_ALGEBRA_EN_UN_CURSO_DE_FORMACION_CONTINUA.

Ramos, E. y González, B. (2011). El Estudio de Clases japonés con uso de Tecnología Inalámbrica. Una experiencia con profesores de matemáticas. Revista lberoamericana (electrónica) de Sistemas, Cibernética e Informática, RISCl, 9(1), 59-64. Recuperado de http://www.iiisci.org/Journal/RISCl.

Reyes-Santander, P. \& Ramos-Rodríguez, E. (2015). Learning journals for University students' reflection on Advanced Algebra. Reflective Practice, 16, 623-635. doi: $10.1080 / 14623943.2015 .1064385$ 
Rojas, N., Flores, P., y Ramos, E. (2012). El análisis didáctico como herramienta para identificar conocimiento matemático para la enseñanza en la práctica. En L. Rico, J. L. Lupiáñez y M. Molina (Eds.), Análisis Didáctico en Educación Matemática (pp.191-208) Granada: Departamento de Didáctica de las Matemáticas.

Tigchelaar, A., Melief, K., Rijswijk, M., \& Korthagen F. (2005). Elementos de una posible estructura de aprendizaje reflexivo en la formación inicial y permanente de profesores. Proyecto Aprender de la práctica, Comenius 2.1. Recuperado de http://www.xtec.net/formacio/practica_reflexiva/base/aprendre/comenius_elements. pdf

Schön, D. (1983). La formación de profesionales reflexivos: Hacia un nuevo diseño de la enseñanza y el aprendizaje en las profesiones. Madrid: Paidós.

Stein, M.K., Smith, M., Henningsen, M., \& Silver, E. (2000). Implementing standards-based mathematics instruction: A casebook for professional development. New York: Teachers College Press. 